\title{
Polarization of active Janus particles
}

\author{
Thomas Bickel ${ }^{1}$, Guillermo Zecua ${ }^{2}$, and Alois Würger ${ }^{1}$ \\ ${ }^{1}$ Laboratoire Ondes et Matière d'Aquitaine, Université de Bordeaux 63 CNRS, 33405 Talence, France and \\ ${ }^{2}$ Institut für Theoretische Physik, Universität Leipzig, 04103 Leipzig, Germany
}

\begin{abstract}
We study the collective motion of Janus particles in a temperature or concentration gradient $\nabla \psi$. Because of the torque exerted by an external or self-generated field, the particles align their axis on the gradient $\boldsymbol{\nabla} \psi$. In a swarm of self-driven particles, this polarization enhances the interactiondriven confinement. Self-polarization in a non-uniform laser beam could be used for guiding hot particles along a given trajectory.
\end{abstract}

PACS numbers: 82.70.Dd, 05.70.Ln, 47.70.Fw

PACS numbers:

Swarms of self-propelling birds, fish, or insects show dynamical patterns that arise from the fact that each individual adapts to the state of its neighbors [1. Similar phenomena occur for liquid dispersions of active objects. Thus cells of Escherichia coli move along a chemical gradient generated by their neighbors and form stable spatial structures 2. More recently, self-driven Janus particles (JPs) have been studied as a model active system [3, 4]. It has been shown that the interplay of self-propulsion and rotational diffusion leads to Brownian motion with an enhanced diffusion coefficient [5]-10, and that the particles' chemical activity results in cluster formation [11 13 and oriented motion [14. Guiding a single microswimmer along a given trajectory can be achieved by dynamical feedback [15].

Active colloids have been realized by partly coating silica or polystyrene particles with a metal or carbon layer; self-propulsion arises from non-uniform surface properties such as temperature or chemical activity. An excess temperature $\psi=T$ is induced by heating the metal or carbon cap through absorption of laser light [7, 9, 13, 15] or magnetic fields [10]. Chemical signalling with a molecular solute, $\psi=c$, is achieved by electrocatalysis of hydrogen peroxide at a metal cap [3, 5, 11, 12].

A minimal model for self-driven systems consists in a drift-diffusion equation that was originally designed for motile bacteria with chemotactic interactions [16], and that describes complex spatial structures observed in cell cultures [17. More recently this model was adapted to JPs that aggregate due to the chemical gradient generated by their electrocatalytic activity [11]. For thermally active colloids, similar results were derived from the Smoluchowski equation [18, 19]: Self-propulsion strongly enhances the diffusion term, whereas the drift velocity arises from the gradient field $\nabla \psi$ generated by the neighbor particles; a sufficiently strong attractive drift term may even cause the implosion of a swarm of JPs.

In the present Letter we show that active colloids are polarized by their chemical or thermal interactions 20 . A non-uniform field $\psi$ exerts a viscous torque on the JP, which in turn aligns its symmetry axis along the gradient $\nabla \psi$. Self-propulsion of such oriented JPs adds a novel contribution to the drift velocity, which is dominant for strong driving or large Péclet number and which may

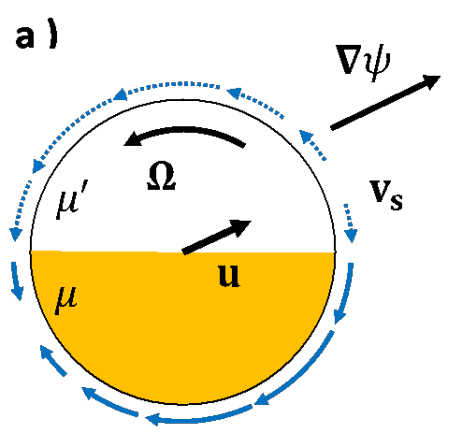

b)
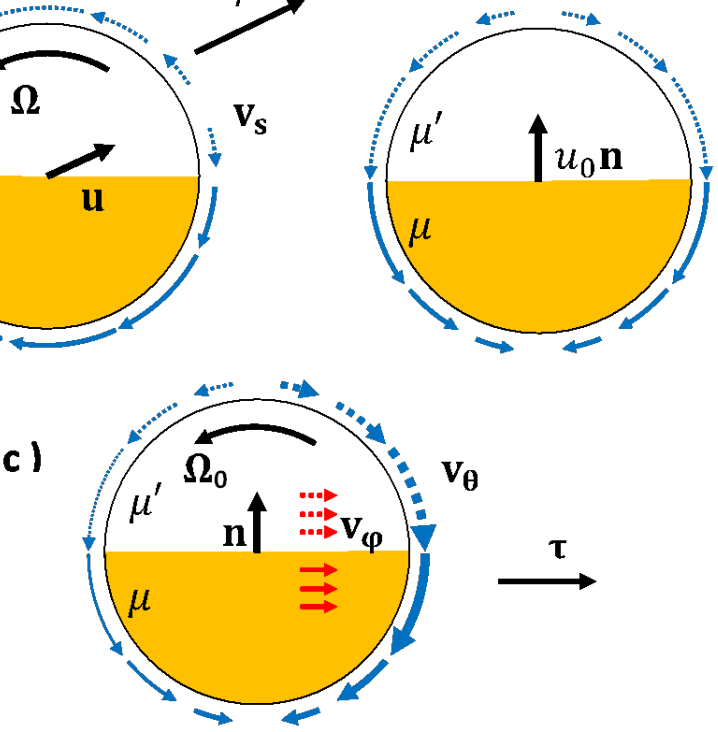

FIG. 1: a) Motion of a Janus particle resulting from an external field gradient $\boldsymbol{\nabla} \psi$. The particle moves at velocity $u \propto \mu^{\prime}+\mu$ along the field gradient and rotates at rate $\Omega \propto \mu^{\prime}-\mu$. The mobility takes the values $\mu^{\prime}$ on the insulating part and $\mu$ on the metal cap; the quasi-slip velocities are indicated by full and dotted arrows. b) Self-propulsion of an active JP. The particle's own field $\psi_{S}$ leads to a quasislip velocity $\mathbf{v}_{s}=\mu \nabla_{\|} \psi_{S}$ that is symmetric with respect to the particle's axis. The particle self-propels at a velocity $u_{0} \propto \mu^{\prime}+\mu$. c) Non-uniform source field with gradient $\boldsymbol{\tau}$. The quasislip velocity at the particle surface has contributions in polar and azimuthal directions; both the magnitude of $\mathbf{v}_{\theta}$ and the orientation of $\mathbf{v}_{\varphi}$ are sensitive to the intensity gradient $\boldsymbol{\tau}$. The resulting angular velocity $\boldsymbol{\Omega}_{0}$ contains terms proportional to $\mu^{\prime} \pm \mu$, as given in (8).

lead to novel collective effects. It turns out that this oriented self-propulsion corresponds to the usual model for bacteria motility.

Polarization of Janus particles. Consider a JP interacting with a concentration or temperature field $\psi$. Within a thin boundary layer, the parallel component of the gradient of the local field $\bar{\psi}$ induces a quasislip 
velocity along the particle surface [22, 23]

$$
\mathbf{v}_{s}(\mathbf{r})=\mu(\mathbf{r}) \nabla_{\|} \bar{\psi}(\mathbf{r}) .
$$

The main result of this paper arise from the materialdependent non-uniform mobility constant $\mu$ and from the properties of the local field $\bar{\psi}$. We consider the case where two values $\mu$ and $\mu^{\prime}$ occur on the two halfspheres of an otherwise homogeneous JP, as indicated in Fig. 1. The resulting quasislip velocities are indicated as solid and dotted lines; their sign and magnitude depend on the mobility values and on the local gradient $\boldsymbol{\nabla}_{\|} \bar{\psi}$.

The quasi-slip velocity (1) constitutes the boundary condition for the velocity field $\mathbf{v}(\mathbf{r})$ of the surrounding fluid, $\left.\mathbf{v}\right|_{B}=\mathbf{u}+\boldsymbol{\Omega} \times \mathbf{r}_{B}+\mathbf{v}_{s}$, where $B$ indicates the outer limit of the interaction layer, typically at a few nanometers from the particle surface. This relation determines the linear and angular velocities of the JP. The former is given by the surface average $\mathbf{u}=-S^{-1} \int d S \mathbf{v}_{s}$, and reads explicitly [24]

$$
\mathbf{u}=-\xi_{1} \frac{\mu+\mu^{\prime}}{3} \nabla \psi(\mathbf{r}) .
$$

Depending on the sign of $\mu+\mu^{\prime}$, the particles move along or opposite to the field gradient. For uniform surface properties $\left(\mu=\mu^{\prime}\right)$ one recovers the usual phoretic velocity of particles in an external field $\boldsymbol{\nabla} \psi[22$.

The factor $\xi_{1}$ in (2) accounts for the deformation of the field $\psi$ due to the presence of the JP. The local gradient is given by the projection on the tangential plane, $\boldsymbol{\nabla}_{\|} \bar{\psi}=\xi_{1}(1-\hat{\mathbf{r}} \hat{\mathbf{r}}) \cdot \boldsymbol{\nabla} \psi$, with the surface normal $\hat{\mathbf{r}}$. For constant $\boldsymbol{\nabla} \psi$ the correction factor reads $\xi_{1}=3 \kappa_{s} /\left(2 \kappa_{s}+\kappa_{p}\right)$; in the case of temperature $\kappa_{s}$ and $\kappa_{p}$ are the heat conductivities of solvant and particle 24]. This form is valid for a sufficiently thin cap, such that the metal layer does not modify the heat flow pattern. In contrast, a thick metal layer results in a spatially varying $\xi(\mathbf{r})$, reducing the slip velocity on the cap and enhancing it on the insulating hemisphere 25]; the limiting case of an isothermal cap is accounted for by putting $\mu=0$ and augmenting $\mu^{\prime}$ by a factor that depends on the orientation of the JP. If $\psi$ is an electric potential, $\kappa_{s}$ and $\kappa_{p}$ are the permittivities; for dielectric materials in water one has $\xi_{1} \approx \frac{3}{2}$ In the case of an applied concentration gradient the $\kappa_{i}$ are the diffusion coefficients; if the solute does not penetrate the particle, one has $\xi=1$; additional effects occur for chemical reactions 21.

A non-uniform mobility factor $\mu(\mathbf{r})$ gives rise to a rotational component of the quasislip velocity [21, 24]. For example, for $\mu^{\prime}=0$ the dotted arrows in Fig. 1a vanish, and the remaining $\mathbf{v}_{s}$ results in a clockwise motion of the surrounding fluid; in order to minimize the viscous stress, the particle then turns in the opposite direction until its axis is parallel to $\boldsymbol{\nabla} \psi$. Equilibrating the surface and viscous forces one obtains the angular frequency

$$
\boldsymbol{\Omega}=-\frac{3}{2 a} \oint \frac{d \mathbf{S} \times \mathbf{v}_{s}}{4 \pi a^{2}},
$$

where $d \mathbf{S}$ is the oriented surface element and $a$ the particle radius. Performing the integral and introducing the unit vector $\mathbf{n}$ along the particle axis, one finds

$$
\boldsymbol{\Omega}=\mathbf{n} \times \mathbf{A}, \quad \mathbf{A}=-\frac{3 \xi_{1}\left(\mu^{\prime}-\mu\right)}{8 a} \boldsymbol{\nabla} \psi(\mathbf{r}) .
$$

Note that the angular velocity is proportional to the mobility difference $\mu-\mu^{\prime}$ of the two hemispheres and thus vanishes for a homogeneous surface. Yet in general $\mu$ and $\mu^{\prime}$ are quite different from each other. If the heated metal cap forms an isotherm, its thermophoretic mobility $\mu$ is zero [25], whereas $\mu^{\prime}$ may take either sign depending on the precise driving mechanism 26, 27. On the other hand, an electrocatalytic mechanism is described finite $\mu$ at the $\mathrm{Pt}$ cap and $\mu^{\prime}=0$ at the remaining surface.

The viscous stress underlying (3), tends to orient the JP along the external field, whereas rotational diffusion with coefficient $D_{r}$ favors dispersion. The resulting kinetics are described by the Smoluchowski equation for the distribution function $f(\mathbf{n})$,

$$
\partial_{t} f=-\mathcal{R} \cdot\left(\boldsymbol{\Omega}-D_{r} \mathcal{R}\right) f \equiv \mathcal{L}_{\mathbf{n}} f,
$$

with the rotation operator $\mathcal{R}=\mathbf{n} \times \nabla_{\mathbf{n}}$, and the gradient $\boldsymbol{\nabla}_{\mathbf{n}}$ with respect to the orientation of the JP [18]. This equation is readily solved in terms of the angle $\theta$ between the particle axis and the field gradient, resulting in $\Omega=A \sin \theta$. The corresponding equation for the steady-state, $\left(A \sin \theta+D_{r} \partial_{\theta}\right) f=0$, is readily solved, $f(\theta) \propto e^{\left(A / D_{r}\right) \cos \theta}$. This effective rotational potential aligns the JP axis on the field gradient, with the mean orientation

$$
\mathbf{n}_{\mathrm{eq}}=\left(\operatorname{coth} \frac{A}{D_{r}}-\frac{D_{r}}{A}\right) \frac{\mathbf{A}}{A} .
$$

For micron-size particles in a temperature gradients of the order $\mathrm{K} / \mu \mathrm{m}$, the ratio $A / D_{r}$ may exceed unity, which is confirmed by experiments on thermophoretic traps with $u a / D>1$ [28. In other words, in such systems JPs are perfectly aligned, $n_{\mathrm{eq}}=1$, whereas for weak fields one has $n_{\mathrm{eq}}=A / 3 D_{r}$. Polarization in an external field $\psi_{\text {ext }}$ is illustrated in Fig. 2a).

Self-propelling Janus particles. Now we consider a swarm of active JPs as shown in Fig. 2b). Their motion consists of single-particle and interaction contributions: Each particle self-propels in its own non-uniform field $\psi_{S}$, whereas that of the neighbors, $\psi(\mathbf{r})=\sum_{j} \psi_{j}\left(\mathbf{r}-\mathbf{r}_{j}\right)$, results in the linear and angular velocities (2) and (4).

The self-generated term $\psi_{S}$ arises from the active surface property $Q$; for example, the surface temperature $T_{S}$ is modified by laser heating at power $Q=\beta I\left(\mathbf{r}_{0}\right)$, where $I$ is the beam intensity at the particle position $\mathbf{r}_{0}$, and $\beta$ the absorption coefficient per unit area of the cap. For constant power $Q_{0}$, the quasislip velocity is symmetric about the particle axis, as illustrated in Fig. 1b, and results in self-propulsion at a speed $\mathbf{n} u_{0}=S^{-1} \oint d S \mu(\mathbf{r}) \boldsymbol{\nabla}_{\|} \psi_{S}$. Solving the diffusion equation for $\psi_{S}$ one finds [24]

$$
u_{0}=\xi_{1} \frac{\mu+\mu^{\prime}}{3} \frac{Q_{0}}{8 \kappa_{s}},
$$



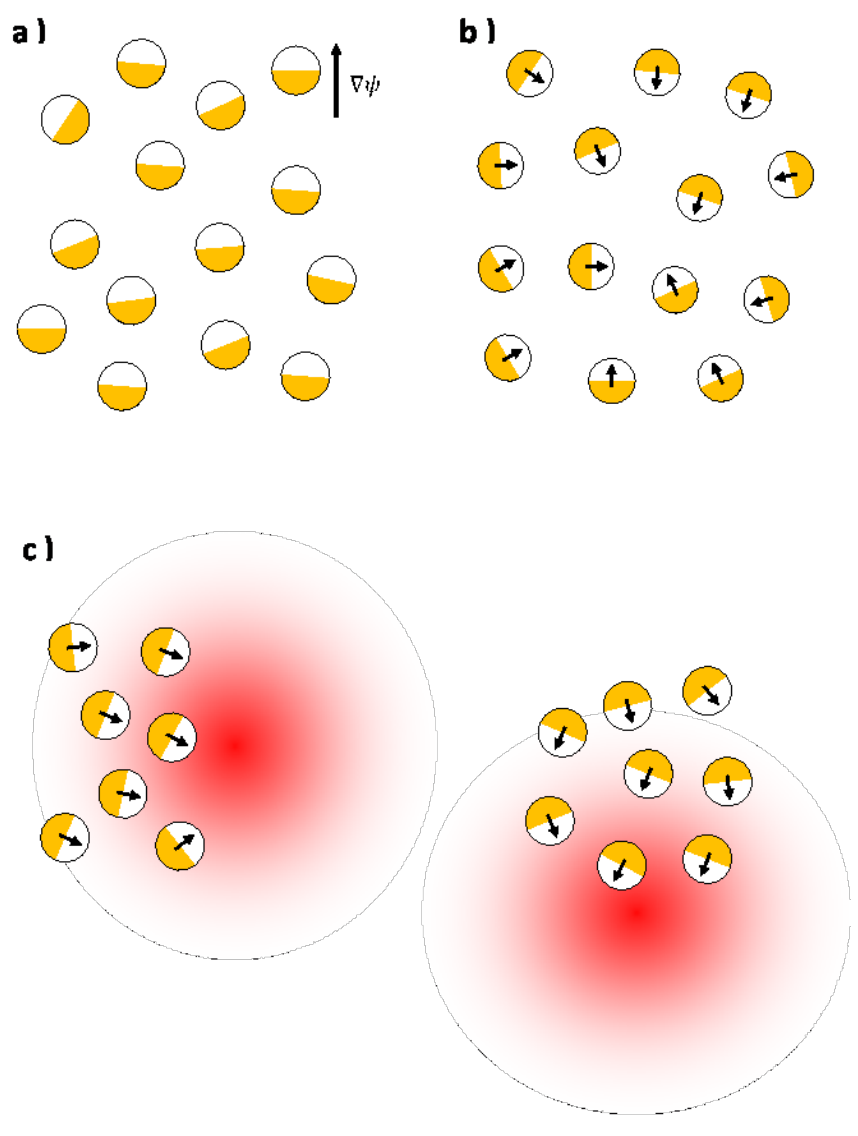

FIG. 2: Polarization of Janus particles in a field gradient $\nabla \psi$. a) Case of an externally applied field $\psi$; the orientation of the particles is given by Eq. (6). There is no self-propulsion, $u_{0}=0$; the small translational velocity $\mathbf{u}$ is not indicated. b) Self-confinement of a swarm of Janus particles. With an appropriate choice of the mobilities $\mu$ and $\mu^{\prime}$, the drift velocity (13) points toward the center of the swarm, thus favoring cluster formation or even implosion 18. The polarization is due to the interaction field $\psi$ which in the simplest case has radial symmetry and its related to the density by $\nabla^{2} \psi+k \rho=$ 0 . c) Guided self-propulsion. Self-driven hot JPs are polarized either by the beam intensity gradient $\tau$ of the heating laser, or by an external temperature gradient $\nabla T_{\text {ext }}$ that results from local heating of the solvant with an IR laser. The JPs follow the heated spot, first to the right and then downward in the figure.

where $Q_{0} / \kappa_{s}$ gives the mean gradient of $\psi_{S}$.

For a spatially varying laser intensity $I(\mathbf{r})$ (or concentration of a catalytic agent [21]), the source field breaks the axial symmetry. The resulting slip velocity has both polar and azimuthal components, as illustrated in Fig. $1 c)$, and exerts a viscous torque

$$
\Omega_{0}=\mathbf{n} \times \mathbf{A}_{0}
$$

which is perpendicular on the particle axis $\mathbf{n}$ and on the gradient of the source, $\boldsymbol{\tau}=\boldsymbol{\nabla} \ln I\left(\mathbf{r}_{0}\right)$,

$$
\mathbf{A}_{0}=-\boldsymbol{\tau}\left(\left(\mu^{\prime}-\mu\right) \frac{5}{18} \xi_{1}+\left(\mu^{\prime}+\mu\right) \widetilde{\xi}\right) \frac{3 Q_{0}}{8 \kappa_{s}}
$$

The first term involves the mobility difference and the dipolar deformation factor $\xi_{1}$. The second one, proportional to $\mu^{\prime}+\mu$, arises from even-order multipoles of $\psi_{S}$ and carries a correction factor $\widetilde{\xi}$ which is given by the weighted average of the diffusivity contrast factors $\xi_{n}=(2 n+1) /\left(n+1+n \kappa_{p} / \kappa_{s}\right)$ [24]. For $\mu^{\prime}>\mu$ both terms result in counterclockwise rotation as shown in Fig. 1c.) The case of catalytic activity is discussed in [21.

Comparing self-propulsed motion with driving due to neighbors in a swarm of JPs, we find that for a colloidal volume fraction of at most a few percent, the velocity $u_{0}$ is much larger than that due to an external field, $u$. The torques exerted by the field of an active neighbor at distance $R$ and by a intensity gradient vary as $\Omega \sim$ $u_{0} a / R^{2}$ and $\Omega_{0} \sim u_{0} \tau$, respectively; depending on the system parameters, one or the other may dominate.

The state of a given JP is described by its position $\mathbf{r}$ and the orientation of its axis $\mathbf{n}$. The distribution function $P(\mathbf{r}, \mathbf{n})$ obeys the equation

$$
\partial_{t} P=-\boldsymbol{\nabla} \cdot\left(u_{0} \mathbf{n}+\mathbf{u}-D \boldsymbol{\nabla}\right) P+\mathcal{L}_{\mathbf{n}} P .
$$

The first term on the right-hand side describes translational motion with velocity $u_{0} \mathbf{n}+\mathbf{u}$ and gradient diffusion with Einstein coefficient $D$. The second term accounts for rotational motion according to (5); the diffusion coefficients are related through $D=\frac{4}{3} a^{2} D_{r}$.

An approximate solution of $(9)$ is obtained by inserting the moment expansion $P(\mathbf{r}, \mathbf{n})=\rho(\mathbf{r})+\mathbf{n} \cdot \mathbf{p}(\mathbf{r})+\ldots$, integrating over $\mathbf{n}$, and truncating the resulting hierarchy at finite order. Following Golestanian [18, we neglect quadrupolar contributions and other small terms in the equation for the polarization vector $\mathbf{p}(\mathbf{r})=$ $(1 / 4 \pi) \int d \mathbf{n n} P$, and thus find 24 ]

$$
\mathbf{p}=-\frac{u_{0}}{6 D_{r}} \boldsymbol{\nabla} \rho+\mathbf{n}_{\mathrm{eq}} \rho, \quad \mathbf{n}_{\mathrm{eq}}=\frac{\mathbf{A}+\mathbf{A}_{0}}{3 D_{r}} .
$$

The first term, which has been derived in previous work [18, 29], accounts for the diffusive transport of polarization in a non-uniform density; the prefactor $u_{0} / D_{r}$ gives the distance over which the particle self-propels during its rotational relaxation time; with $u_{0} \sim 10 \mu \mathrm{m} / \mathrm{sec}$ and $1 / D_{r} \sim 1$ sec one finds about 10 microns. The second term $\mathbf{n}_{\text {eq }} \rho$ accounts for active polarization of JPs. Fig. 2b) illustrates the alignement on the field gradient (4) created by nearby JPs, and Fig. 2c) shows selfpolarization of the JPs along the gradient (8).

With the polarization $\mathbf{p}$ one obtains the drift-diffusion equation for the density $\rho$ 24]

$$
\partial_{t} \rho=-\nabla \cdot\left(\mathbf{u}_{\text {eff }} \rho-D_{\text {eff }} \boldsymbol{\nabla} \rho\right),
$$

where $D_{\text {eff }}=D\left(1+\frac{2}{9} \mathrm{Pe}^{2}\right)$ is the effective diffusion coefficient and $\mathrm{Pe}=u_{0} a / D$ the Péclet number [4, 5]. The effective velocity

$$
\mathbf{u}_{\text {eff }}=\mathbf{u}+u_{0} \mathbf{n}_{\text {eq }}
$$

consists of the interaction-driven drift $(2)$ and oriented self-propulsion with the equilibrium polarization $\mathbf{n}_{\mathrm{eq}}=$ $\left(\mathbf{A}+\mathbf{A}_{0}\right) / 3 D_{r}$. 
In a constant source field $Q$ there is no selfpolarization, $\mathbf{A}_{0}=0$, and the drift velocity can be cast in the form

$$
\mathbf{u}_{\mathrm{eff}}=\xi\left(-\frac{\mu+\mu^{\prime}}{3}+\mathrm{Pe} \frac{\mu-\mu^{\prime}}{6}\right) \nabla \psi(\mathbf{r}) .
$$

The first term in parentheses, which has derived previously [11, 18, is independent of the particle orientation. The second one has not been considered so far; it arises from self-propulsion of polarized JP and dominates at large Péclet number. Since $\operatorname{Pe} \propto \mu+\mu^{\prime}$, the two terms in (13) carry opposite signs for $\mu>\mu^{\prime}$.

Temperature and concentration fields generated by the JPs' heat absorption or chemical activity, satisfy $\nabla^{2} \psi+k \rho=0$ with the particle density as source term. Then the sign of the prefactor of $\mathbf{u}_{\text {eff }}$ determines whether self-propulsion disperses or confines a cloud of JPs. A sufficiently large negative drift velocity results in clustering as illustrated in Fig. 2b [11-13] and may even drive implosion of the swarm [18]. This latter scenario has been discussed in detail for $\mathbf{u}_{\text {eff }}=\mathbf{u}$ and $\mu=\mu^{\prime}<0$, corresponding to a negative Soret coefficient [18. The correction term $u_{0} \mathbf{n}_{\text {eq }}$ derived here, is dominant for $|\mathrm{Pe}|>0$ and, according to 13 results in attraction $\mu^{\prime 2}>\mu^{2}$, independently of the sign of the mobilities. Thus polarizaton enhances $\mathbf{u}_{\text {eff }}$ by a factor Pe and, at large Péclet number, even modifies the dynamical phase diagram. Experiments on cluster formation [11-13] and oriented motion 14 support the qualitative features of the drift-diffusion model, yet available data are not sufficiently precise for a quantitative comparison.

Guided self-propulsion. So far we discussed polarization along the field gradient $\nabla \psi$ generated by the heat absorption or chemical activity of neighbor JPs. Here we discuss the case where both propulsion and polarization result from the particle's self-generated temperature field $T_{S}$. With the linear velocity $u_{0}$ and the order parameter $\mathbf{n}_{\text {eq }}=\mathbf{A}_{0} / 3 D_{r}$, we obtain oriented motion along the intensity gradient of the laser beam,

$$
\mathbf{u}_{\mathrm{eff}}=u_{0} \mathbf{n}_{\mathrm{eq}}=\frac{4}{9} \operatorname{Pe} a \mathbf{A}_{0} .
$$

Note that this a single-particle property and varies with the square of the laser intensity. A physical realization is sketched in Fig. 2c, where a focussed laser beam illuminates a swarm of JPs. Since the particles move towards the center of the beam according to (3), they could be guided by a mobile laser beam along a given trajectory.

Chemotaxis of bacteria. We compare the motion of polarized JPs with bacteria that are guided by chemotactic signalling. E. coli self-propels through flagella rotating in the "run" mode at a velocity $u_{0}$ along its axis $\mathbf{n}[17$. After a period of $\tau \sim 1 \mathrm{sec}$, they switch to the "tumble" mode, which randomly changes the orientation and thus plays the role of rotational diffusion. The cell performs a random walk with diffusion coefficient $D_{\text {eff }} \sim u_{0}^{2} \tau$.

Bacteria are not able to actively reorient in a field gradient, contrary to JPs according to (3). Yet they are sensitive to the concentration of certain solutes. If a cell detects a favorable change of $\psi$ along its trajectory, it augments the time $\tau$; on the other hand, if it feels it goes the wrong direction, it switches more rapidly to the tumble mode. As a consequence, the bacterium spends more time in an orientation toward the source [17. Assuming a linear variation with the concentration gradient, one has $\tau=\tau_{0}+\alpha \mathbf{n} \cdot \nabla \psi$, where $\alpha$ describes the strength of the response to chemical signalling. The resulting polarization $\mathbf{n}_{\mathrm{eq}}=\frac{1}{3} \alpha \boldsymbol{\nabla} \psi$ results in the drift velocity

$$
\mathbf{u}_{\mathrm{eff}}=u_{0} \mathbf{n}_{\mathrm{eq}}=\frac{u_{0}}{3} \alpha \nabla \psi
$$

Comparison with the drift velocity of JPs shows that bacteria motion corresponds to the second term in Eq. (12), that is, to self-propulsion along the fieldd gradient $\nabla \psi$.

In view of Eqs.12 and (15) one expects for swarms of JPs a dynamical behavior very similar to that observed in bacteria cultures. Fine-tuning of the surface parameters $\mu$ and $\mu^{\prime}$ would allow to separate the effects of phoretic motion $\mathbf{u}$ and of oriented self-propulsion $u_{0} \mathbf{n}_{\text {eq }}$. Since only the latter is present in $(15)$, the relative weight of these terms is an important parameter when comparing the motion of JPs and bacteria.

We conclude with a remark on hydrodynamic interactions which have been neglected in the present paper. The interactions considered here are mediated by thermal or concentration gradients $\boldsymbol{\nabla} \psi$ which in three dimensions vary with the square of the inverse distance, $\nabla \psi \propto r^{-2}$. Depending on the symmetry of quasi-slip velocity, hydrodynamic interactions decay as $r^{-3}$ or $r^{-2}$ [25, 30, 31]; the latter term may attain values comparable to the interaction contribution $\mathbf{u}$ in 12 . Yet at large Péclet number, it is small as compared to the self-propulsion contribution $u_{0} \mathbf{n}_{\mathrm{eq}}$.

Acknowledgment. A.W. acknowledges support through the Leibniz program of Universität Leipzig during the summer term 2013, and thanks the groups of Frank Cichos and Klaus Kroy for their kind hospitality.
[1] T. Vicsek, A. Zafeiris, Phys. Rep. 517, 71 (2012)

[2] J. Adler, Science 153, 708 (1966)

[3] W.F. Paxton, A. Sen, and T.E. Mallouk, Chem. Eur. J. 11, 6462 (2005).
[4] R. Golestanian, T.B. Liverpool, and A. Ajdari, Phys. Rev. Lett. 94, 220801 (2005)

[5] J.R. Howse, R.A.L. Jones, A.J. Ryan, T. Gough, R. Vafabakhsh, and R. Golestanian, Phys. Rev. Lett. 99, 
$048102(2007)$

[6] J. Palacci, C. Cottin-Bizonne, C. Ybert, L. Bocquet, Phys. Rev. Lett. 105, 088304 (2010)

[7] H.-R. Jiang, N. Yoshinaga, M. Sano, Phys. Rev. Lett. 105, 268302 (2010)

[8] G. Volpe, I. Buttinoni, D. Vogt, H.-J. Kümmerer, and C. Bechinger, Soft Matter 7, 8810 (2011)

[9] I. Buttinoni, G. Volpe, F. Kümmel, G. Volpe, C. Bechinger, J. Phys.: Cond. Mat. 24, 284129 (2012)

[10] L. Baraban, R. Streubel, D. Makarov, L., D. Karnaushenko, O.G. Schmidt, G. Cuniberti, ACS Nano 7, 1360 (2013)

[11] I. Theurkauff, C. Cottin-Bizonne, J. Palacci, C. Ybert, L. Bocquet, Phys. Rev. Lett. 108, 268303 (2012)

[12] J. Palacci, S. Sacanna, A.P. Steinberg, D.J. Pine, P.M. Chaikin, Science 339, 936 (2013)

[13] I. Buttinoni, J. Bialke, F. Kümmel, H. Löwen, C. Bechinger, T. Speck, Phys. Rev. Lett. 110, 238301 (2013)

[14] L. Baraban, S.M. Harazim, S. Sanchez, O.G. Schmidt, Angew. Chem. Int. Ed. 52, 5552 (2013)

[15] B. Qian, D. Montiel, A. Bregulla, F. Cichos, H. Yang, Chem. Sci. 4, 1420 (2013)

[16] E.F. Keller, L.A. Segel, J. Theor. Biol. 26, 399 (1970)

[17] M.P. Brenner, L.S. Levitov, E.O. Budrene, Biophys. J. 74, 1677 (1998)
[18] R. Golestanian, Phys. Rev. Lett. 108, 038303 (2012)

[19] J.A. Cohen, R. Golestanian, arXiv:1309.3318v1 (2013)

[20] After submission of this paper we became aware of the preprint 21] that discusses a mechanism similar to ours.

[21] S. Saha, R. Golestanian, S. Ramaswamy, arXiv:1309.4947 2 (2013)

[22] J.L. Anderson, Ann. Rev. Fluid Mech. 21, 61 (1989)

[23] R. Golestanian, T.B. Liverpool, and A. Ajdari, New J. Phys. 9, 126 (2007)

[24] The supplementary material file gives the derivation of the local field gradient, and the translationial and angular velocities.

[25] T. Bickel, A. Majee, and A. Würger, Phys. Rev. E 88, 012301 (2013)

[26] D. Vigolo, S. Buzzaccaro and R. Piazza, Langmuir, 26, $7792(2010)$

[27] A. Würger, Rep. Prog. Phys., 73, 126601 (2010)

[28] R. Di Leonardo, F. Ianni, G. Ruocco, Langmuir 25, 4247 (2009)

[29] M. E. Cates and J. Tailleur, Europhys. Lett. 101, 20010 (2013)

[30] I. Llopis, I. Pagonabarraga, J. Non-Newtonian Fluid Mech. 165, 946 (2010)

[31] A. Zöttl, H. Stark, arXiv:1309.4352 1 (2013) 